\title{
Heterologous expression of the isopimaric acid pathway in Nicotiana benthamiana and the effect of N-terminal modifications of the involved cytochrome P450 enzyme
}

Thiyagarajan Gnanasekaran ${ }^{1 \dagger}$, Konstantinos Vavitsas $^{1 \dagger}$, Johan Andersen-Ranberg ${ }^{1,2}$, Agnieszka Zygadlo Nielsen ${ }^{1}$, Carl Erik Olsen ${ }^{1}$, Björn Hamberger ${ }^{1}$ and Poul Erik Jensen ${ }^{1 *}$

\begin{abstract}
Background: Plant terpenoids are known for their diversity, stereochemical complexity, and their commercial interest as pharmaceuticals, food additives, and cosmetics. Developing biotechnology approaches for the production of these compounds in heterologous hosts can increase their market availability, reduce their cost, and provide sustainable production platforms. In this context, we aimed at producing the antimicrobial diterpenoid isopimaric acid from Sitka spruce. Isopimaric acid is synthesized using geranylgeranyl diphosphate as a precursor molecule that is cyclized by a diterpene synthase in the chloroplast and subsequently oxidized by a cytochrome P450, CYP720B4.

Results: We transiently expressed the isopimaric acid pathway in Nicotiana benthamiana leaves and enhanced its productivity by the expression of two rate-limiting steps in the pathway (providing the general precursor of diterpenes). This co-expression resulted in 3-fold increase in the accumulation of both isopimaradiene and isopimaric acid detected using GC-MS and LC-MS methodology. We also showed that modifying or deleting the transmembrane helix of CYP720B4 does not alter the enzyme activity and led to successful accumulation of isopimaric acid in the infiltrated leaves. Furthermore, we demonstrated that a modified membrane anchor is a prerequisite for a functional CYP720B4 enzyme when the chloroplast targeting peptide is added. We report the accumulation of $45-55 \mu \mathrm{g} / \mathrm{g}$ plant dry weight of isopimaric acid four days after the infiltration with the modified enzymes.
\end{abstract}

Conclusions: It is possible to localize a diterpenoid pathway from spruce fully within the chloroplast of $\mathrm{N}$. benthamiana and a few modifications of the N-terminal sequences of the CYP720B4 can facilitate the expression of plant P450s in the plastids. The coupling of terpene biosynthesis closer to photosynthesis paves the way for light-driven biosynthesis of valuable terpenoids.

Keywords: Chloroplasts, Cytochrome P450, Diterpenoids, Isopimaric acid, Protein engineering

\footnotetext{
* Correspondence: peje@plen.ku.dk

${ }^{\dagger}$ Equal contributors

'Department of Plant and Environmental Sciences, Copenhagen Plant Science Centre, UNIK Center for Synthetic Biology, Villum Research Center

"Plant Plasticity", University of Copenhagen, Thorvaldsensvej 40, DK-1871

Frederiksberg C, Copenhagen, Denmark

Full list of author information is available at the end of the article
} 


\section{Background}

Plant specialized metabolites comprise a large pool of compounds that play a major role in functions such as interaction with the environment, defense, and stress tolerance [1]. Diterpenoids- $\mathrm{C}$-20 terpenes that number more than 12,000 identified metabolites [2] - represent a considerable part of this chemical wealth. In addition, they have a wide range of applications as pharmaceuticals, cosmetics, and food additives [3-6]. In plants, the initial steps of the diterpenoid biosynthesis occur in the plastids, where diterpene synthases (diTPS) catalyze the cyclization of geranylgeranyl pyrophosphate (GGPP) [5, 7]. Diterpenoids display a remarkable diversity in terms of structure and physiological role; this chemical variety initiates from the amount of carbon skeleton arrangements the diTPS can produce $[2,7]$, and which is further augmented by cytochrome P450s [8].

Cytochrome P450s are a versatile enzyme superfamily that performs stereo-specific oxygenation via an electronconsuming, heme-dependent catalytic mechanism [9]. In plants, the majority of the P450s are found in the endoplasmic reticulum (ER) anchored via their N-terminal membrane domain, although some P450s are found soluble in the mitochondria and chloroplasts [10, 11]. Expressing a plant $\mathrm{P} 450$ in bacteria or yeast is not straightforward, due to issues such as codon usage and membrane localization of the protein [12]. Another limiting factor is the availability of reducing power in heterologous hosts $[3,13]$. Recently, it was shown that plant P450s can use electrons directly from photoreduced ferredoxin, both in vitro and in vivo [14-19].

Isopimaric acid is a diterpene resin acid produced by conifers. It is a defense compound that prevents spore germination of the pathogenic fungus Ophiostoma ips and affects the feeding habits of gypsy moth (Lymantria dispar) larvae and various sawfly species [20-22]. Isopimaric acid is also reported to have antibacterial activity against multidrug resistant Staphylococcus aureus strains that can cause bacteremia, endocarditis and hemolytic pneumonia in humans [23]. In Sitka spruce, the isopimaric acid pathway involves only two enzymes, a bi-functional diterpene synthase, isopimara-7, 15-diene synthase (diTPS-ISO) and a multifunctional cytochrome P450, CYP720B4. The diterpene synthase converts GGPP to the tricyclic diterpene isopimara-7, 15-diene (isopimaradiene). In three subsequent oxygenation reactions, the ERlocalized CYP720B4 catalyzes the conversion of isopimaradiene into isopimaric acid (Fig. 1) [24-26].

In this work, we studied the expression of the isopimaric acid pathway in Nicotiana benthamiana leaves using transient infiltration with Agrobacterium tumefaciens. Plant chloroplasts are attractive bioengineering targets: they already serve as biosynthetic sites for various metabolites, they provide a prokaryotic-like environment in terms of protein expression and regulation, and they offer the opportunity to directly exploit the reducing power of photosynthesis [3, 27, 28]. We modified the N-terminal part of the CYP720B4 in order to target it to the chloroplast and we assessed our engineering approaches via immunoblotting and metabolite analysis.

\section{Results and discussion \\ In-vivo production of isopimaradiene and isopimaric acid in $N$. benthamiana}

To express the entire isopimaric acid pathway, we infiltrated $N$. benthamiana plants with A. tumefaciens, harboring plasmids encoding both diTPS-ISO and CYP720B4. P19 silencing repressor gene-infiltrated plants [29] were used as controls. The expressed diTPS-ISO is active as confirmed by the formation of isopimaradiene in the infiltrated leaves (Fig. 2a, b) (for expression of the diTPS-ISO protein in the infiltrated leaves refer to the following section). Upon coexpression with CYP720B4, isopimaradiene was depleted and isopimaric acid accumulated (Fig. 2c, d), indicating efficient conversion. The P19 blank infiltration samples displayed a background free of isopimaradiene and isopimaric acid.

To improve the production of isopimaric acid, we utilized the 1-deoxyxylulose-5-phosphate synthase (DXS) and GGPP synthase (GGPPs) from Plectranthus barbatus (Indian coleus). Those two enzymes belong to the GGPP biosynthetic pathway and are considered rate-limiting for diterpenoid production [30, 31]. The co-expression of DXS and GGPPs together with the isopimaric acid enzymes led to a 3-fold increase in the accumulation of both isopimaradiene and isopimaric acid (Fig. 2a, c). We overexpressed the enzymes of $P$. barbatus, instead of the native ones, in order to avoid any feedback regulation and post- translational modifications that adjust the DXS and GGPPs activity [32-34]. In a previous study from our lab (Randberg et al., unpublished), we showed that expressing DXS and GGPPs from P. barbatus in $N$. benthamiana were efficient in boosting diterpenoid levels compared to co-expression of the native DXS and GGPPs genes, which had no effect on boosting diterpenoid level.

Rerouting GGPP into novel diterpenes might have an effect on native GGPP-derived metabolites or pigments. We measured the relative quantity of the photosynthetic pigments chlorophyll $a$, chlorophyll $b$, violaxanthin, neoxanthin, lutein, and $\beta$-carotene by performing high performance liquid chromatography (HPLC). Comparing infiltrated leaf extracts and controls showed no detectable difference (Fig. 3). 


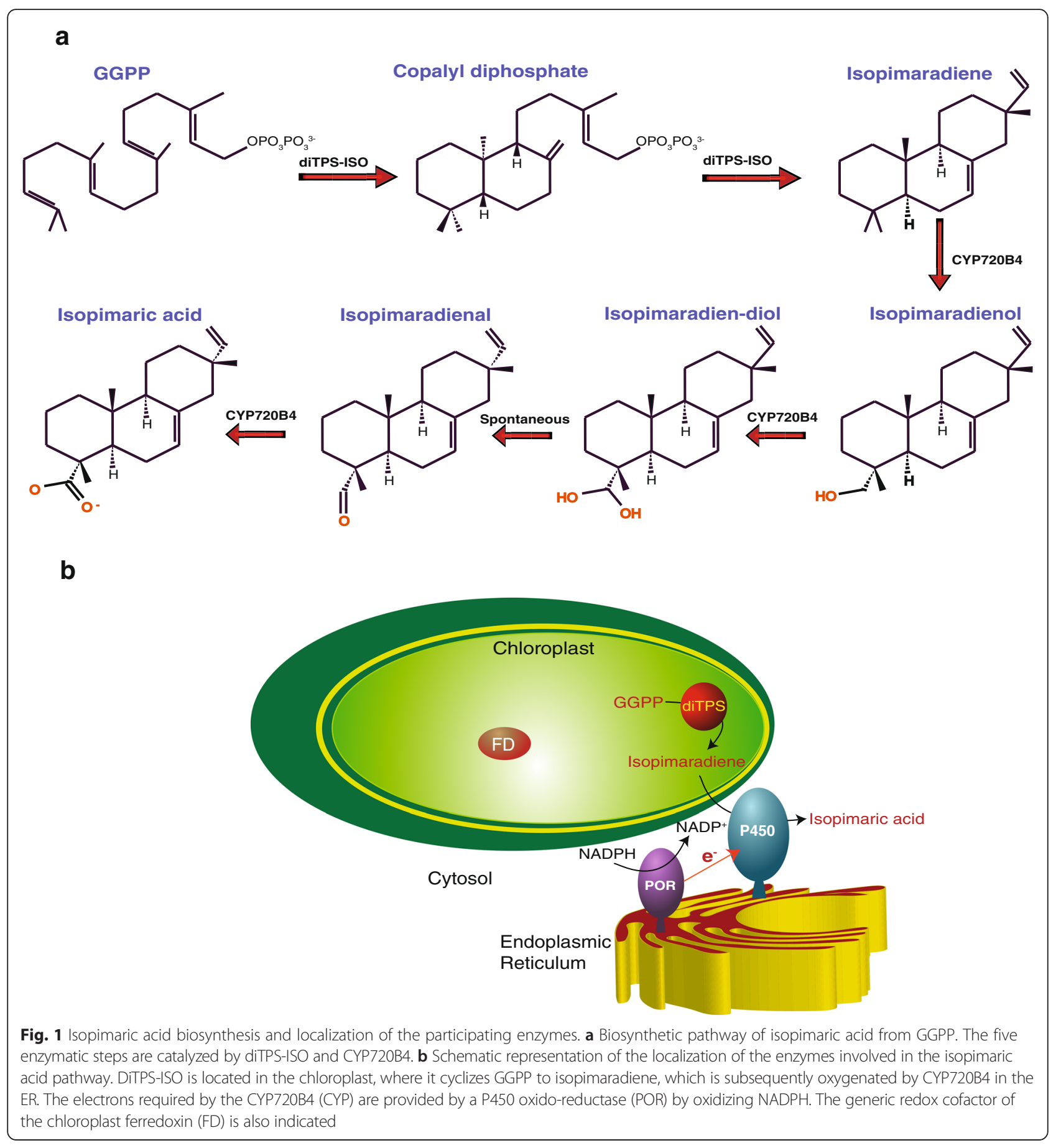

\section{Expression of $\mathrm{N}$-terminal modified CYP720B4 in $\mathrm{N}$. benthamiana}

To express CYP720B4 in $N$. benthamiana, we used the native CYP720B4 coding sequence and two different CYP720B4 variants that were modified in the $\mathrm{N}$ terminal membrane anchor domain (Fig. 4a). Initially, to investigate if the native membrane anchor of CYP720B4 is essential for its activity, we eliminated the $\mathrm{N}$-terminal membrane domain of CYP720B4 ( $\Delta$ Tm-CYP720B4) by truncating the region that encodes the first 37 amino acids (Fig. 4b). We also implemented a strategy similar to the one used to express the human P450 17-alpha hydroxylase [35] and CYP720B4 [26] in Escherichia coli: we deleted $7 \mathrm{~N}$-terminal amino acids from the membrane anchor $(\Delta 3-9)$ and mutated a valine in position 13 to alanine (Barnes-CYP720B4) (Fig. 4b). Finally, to target the CYP720B4 enzyme to the chloroplasts, we made the following gene constructs variants Tp-CYP720B4, Tp- 

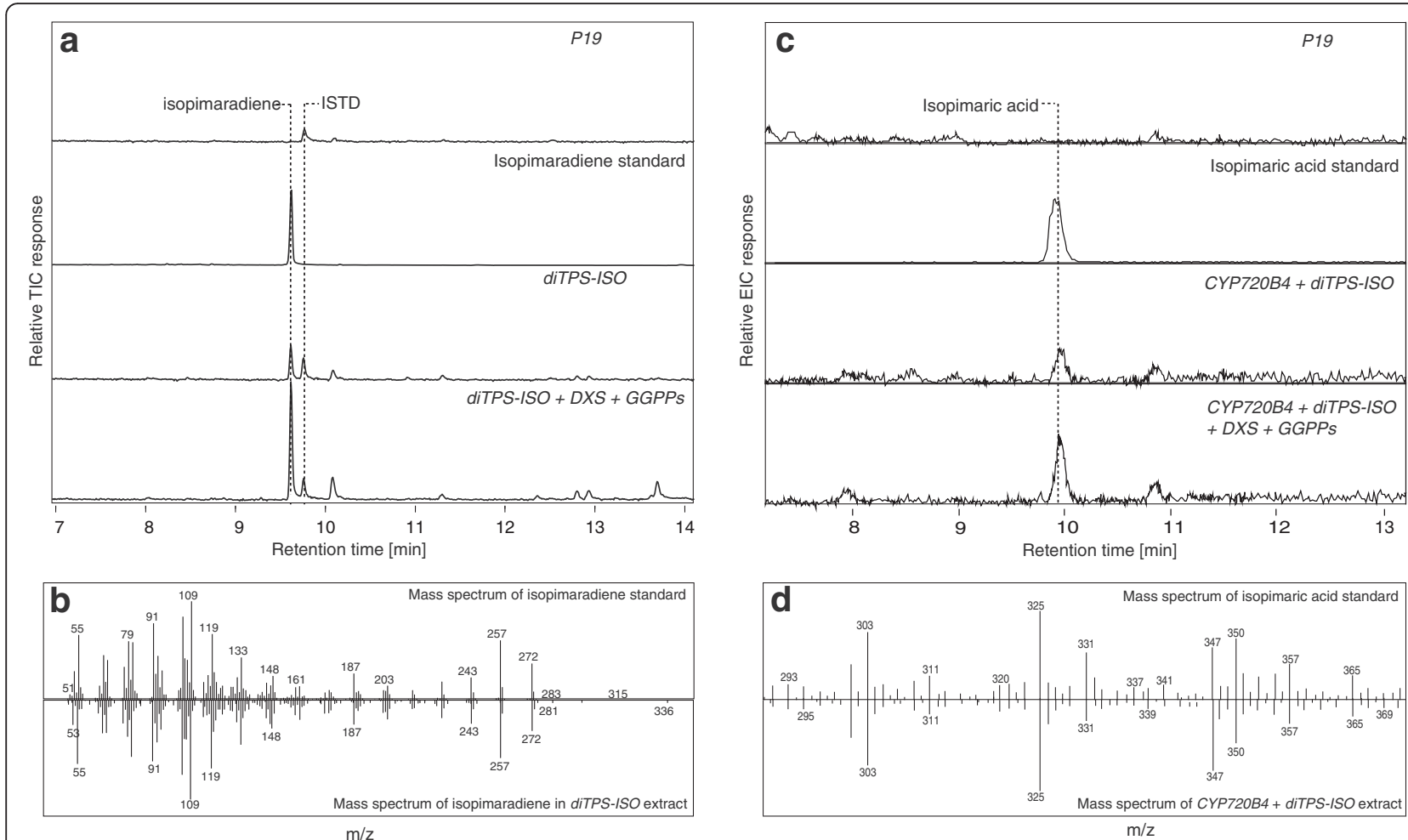

Fig. 2 Metabolite analysis of infiltrated plant leaves. a Total lon Chromatograms (TIC) for the detection of isopimaradiene using GC-MS analysis. Samples were spiked with the internal standard Eicosene (ISTD). b Mass spectra of isopimaradiene detected in the standard and diTPS-ISO infiltrated leaf extracts. c Extracted ion chromatograms $\left(\mathrm{m} / \mathrm{z}-303[\mathrm{M}+\mathrm{H}]^{+}, 325[\mathrm{M}+\mathrm{Na}]^{+}, 347[\mathrm{M}+2 \mathrm{Na}]^{+}\right)$for the detection of isopimaric acid using LC-MS analysis. Isopimaric acid is detected at retention time $10 \mathrm{~min}$. $\mathbf{d}$ Mass spectra of isopimaric acid detected in the standard and CYP720B4 + diTPS-ISO infiltrated leaf extracts

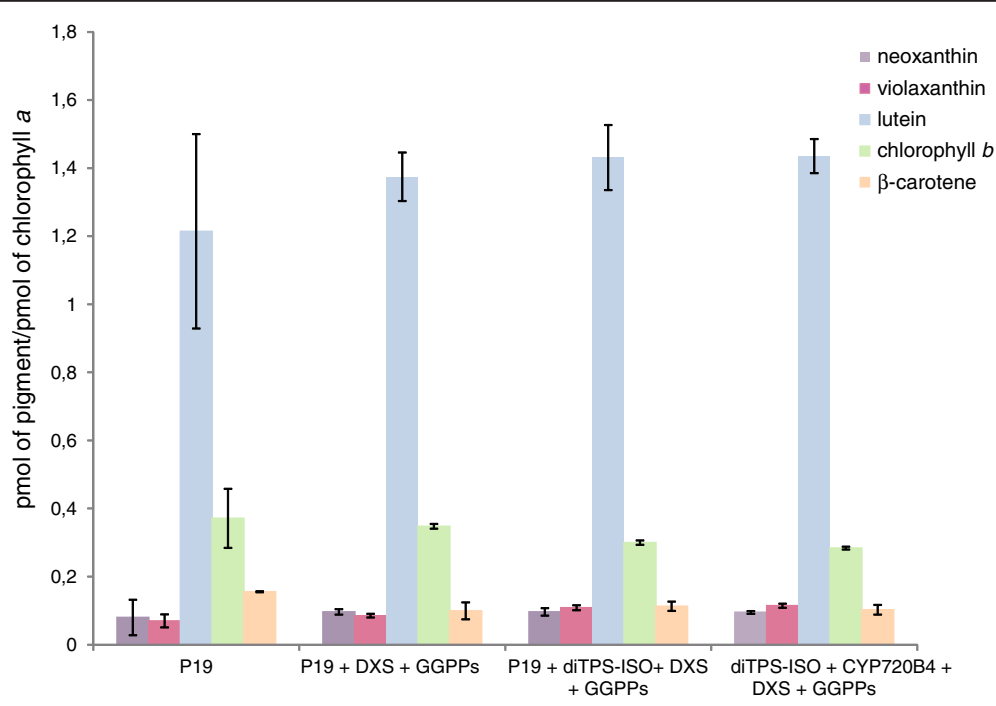

Fig. 3 Pigment analysis of infiltrated leaves. P19-infiltrated plants were used as controls. The increased expression of DXS and GGPPs during the transient expression experiments did not result in significant variations in pigment composition. Pigments quantities are given relative to chlorophyll $a$. Biological triplicates were used for the quantification 


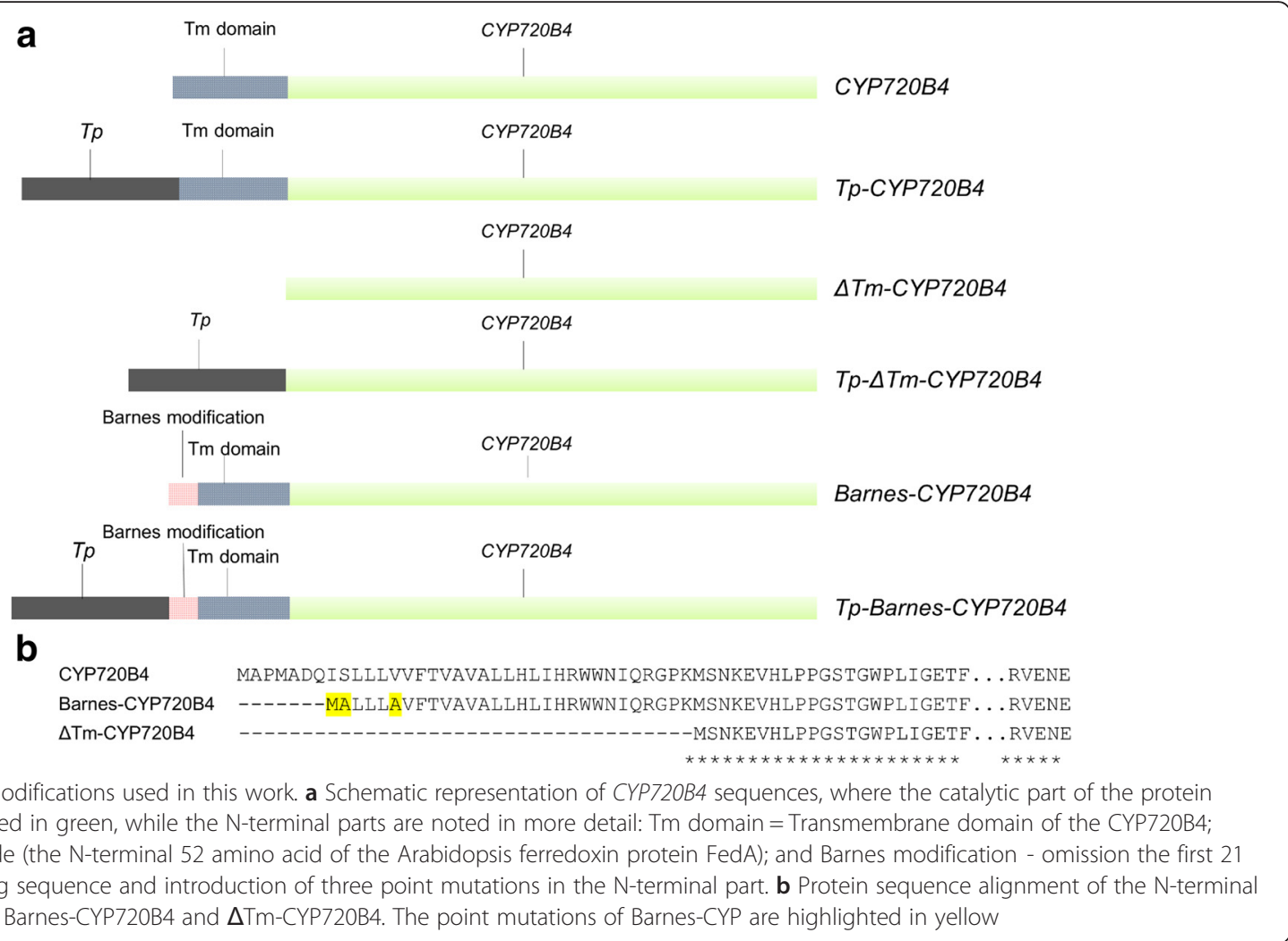

$\triangle T$ m-CYP720B4 and Tp-Barnes-CYP720B4 by fusing the coding region of the chloroplast transit peptide (Tp) of the Arabidopsis thaliana ferredoxin protein FedA [36] in the N-termini of CYP720B4, $\triangle \mathrm{Tm}$-CYP720B4 and Barnes-CYP720B4 (Fig. 4a).

Immunoblots of the crude leaf extracts showed that CYP720B4 was expressed from all CYP720B4 construct variants (Fig. 5). Importantly, the enzymes that contained ferredoxin transit peptide (Tp-Barnes-CYP720B4 and Tp- $\Delta \mathrm{Tm}-\mathrm{CYP720B} 4)$ showed two protein bands, consistent in size with the precursor protein and the mature protein where the Tp was removed. Such a processing takes place during protein translocation into the plastid [37], corroborating the successful targeting of Tp-Barnes-CYP720B4 and Tp- $\Delta \mathrm{Tm}-\mathrm{CYP720B} 4$ to the chloroplast.

\section{Effect of N-terminal modifications in CYP720B4 activity}

The diTPS-ISO and the CYP720B4 are naturally directed to the chloroplast and ER, respectively (Fig. 1b). CYP720B4 remains active even after complete removal of the transmembrane domain (Fig. 6); it is possible that either the remaining enzyme core can direct itself to the ER or that interaction with the P450 oxidoreductase allows proper localization. Truncated P450s do not generally lose the ability to associate with lipid membranes, however the ratio between ER-bound and soluble protein can change in favor of the latter [10, 38]. Barnes-CYP720B4 activity does not seem to deteriorate after the N-terminal modification (Fig. 6).

Most chloroplast proteins are encoded by the nucleus, hence they need to move into the organelle posttranslationally [27]. Previously, CYP79A1 and CYP71E1, involved in the dhurrin biosynthetic pathway, have been functionally expressed in tobacco chloroplasts by fusing the N-terminus of the enzyme with the ferredoxin transit peptide [15]. We used the same approach in the present work, but the native CYP720B4 lost enzymatic activity when fused with the transit peptide (Fig. 6). In the dhurrin study [15], the P450 substrate was the hydrophilic compound tyrosine, while CYP720B4 acts on the hydrophobic isopimaradiene, which is a cyclic and non-oxygenated hydrocarbon (Fig. 1a). Taking this consideration one step further, we speculate that a proper membrane anchoring is crucial for CYP720B4 activity or substrate availability for the CYP720B4 active site. Membrane-bound and soluble P450s adopt different orientation of the heme within the protein [39], a fact that could result in major differences in the enzymatic properties.

The deletion of the transmembrane domain of CYP720B4 resulted in an active enzyme; however, the addition of the targeting peptide to Tp- $\Delta \mathrm{Tm}-\mathrm{CYP} 720 \mathrm{~B} 4$ led to no product formation, despite the apparent high 

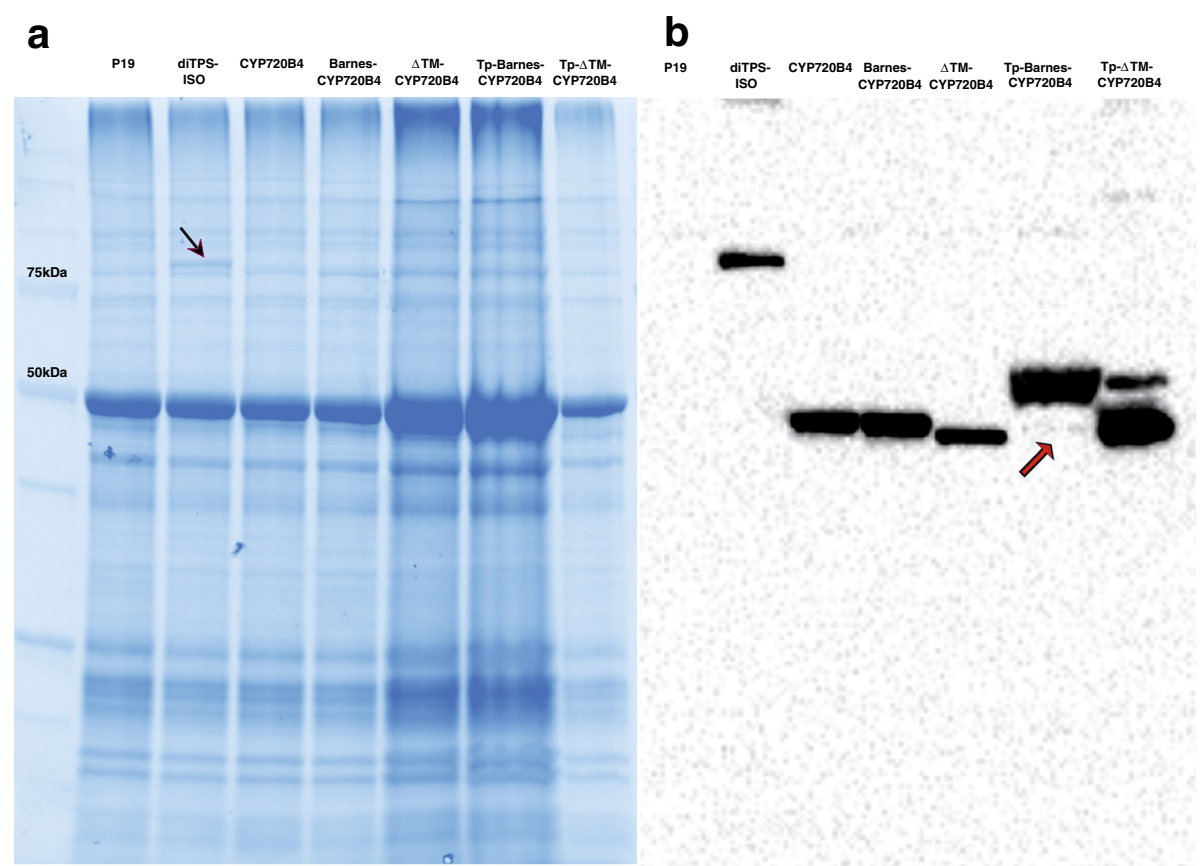

Fig. 5 Analysis of protein expression in the transiently transformed leaves. Shown are SDS-PAGE (a) and immunodetection (b) of total protein

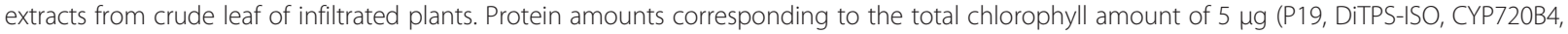
and Barnes-CYP720B4), $10 \mu \mathrm{g}$ ( $\Delta$ Tm-CYP720B4), $15 \mu \mathrm{g}$ (Tp-Barnes-CYP720B4) and $3 \mu \mathrm{g}$ (Tp- $\Delta T \mathrm{Tm}-\mathrm{CYP720B4)}$ were electrophoresed using SDS-PAGE. For immunodetection, Anti-FLAG antibody was used. The expected molecular masses of the FLAG-tag proteins DiTPS-ISO, CYP720B4, Barnes-CYP720B4 and $\triangle$ Tm-CYP720B4 without the chloroplast transit peptide are 92, 55, 54 and $51 \mathrm{kDa}$ respectively, and the masses of Tp-Barnes-CYP720B4 and Tp- $\Delta$ Tm -CYP720B4 are 60 and $57 \mathrm{kDa}$ respectively. The black arrow points to the visible protein band of DiTPS-ISO in SDS-PAGE. Red arrow points to faint immunodetection signal of Tp-Barnes-CYP720B4 that has presumably been processed upon transport into the chloroplasts

amount of processed enzyme detected in immunoblotting (Fig. 5b). In contrast, the Tp-Barnes-CYP720B4 was active. This suggests that the Barnes modification stabilizes CYP720B4 in the chloroplast. The large amount of unprocessed protein detected in the immunoblot could mean that a significant fraction of the enzyme remains in the cytosol or is ectopically inserted in the ER.

\section{Conclusions}

Chloroplasts have already been engineered to overproduce carotenoids [40,41] and have a large biotechnological potential that has been extensively reviewed [42, 43]. In this work, we showed that it is possible to localize a diterpenoid pathway from spruce fully within the chloroplast of $N$. benthamiana. We demonstrate that a few modifications of the $\mathrm{N}$-terminal sequences of the CYP720B4, such as the modifications mentioned by Barnes and coworkers [35], can facilitate the expression of plant P450s not only in bacteria [26], but also in the plastids. Moreover, we assume that the membrane anchor is of importance for heterologous expression in algal chloroplasts or cyanobacteria, organisms that grow in liquid cultures and have potential as largescale production systems [44].
P450s display various degrees of substrate promiscuity [45], a fact that can account for the terpenoid diversity, while it permits combinatorial approaches that differentially oxygenate the same cyclic skeleton [46]. Therefore, P450 enzymes are prominent candidates for synthetic biology exploitation, especially under the consideration that they are involved in the synthesis of commercially important compounds [47-50]. Understanding their heterologous expression properties and enzymatic characteristics can enhance terpenoid productivity and commercial availability via successful metabolic engineering approaches.

\section{Methods}

Plant material and microbial strains

NEB10 $\beta$ Escherichia coli cells (New England Biolabs) were used for all sub-cloning steps. Respective constructs were transformed into chemically competent NEB10 $\beta$ E. coli cells and transformants were selected on $50 \mathrm{~g} / \mathrm{L}$ Kanamycin LB plates. The positive transformants were screened using PCR and grown overnight in $50 \mathrm{~g} / \mathrm{L}$ kanamycin LB medium. Plasmid DNA was isolated using the QIAprep Spin $^{\text {mi }}$ miniprep Kit (Qiagen) according to provider's manual. A. tumefaciens PGV 3850 cells were 

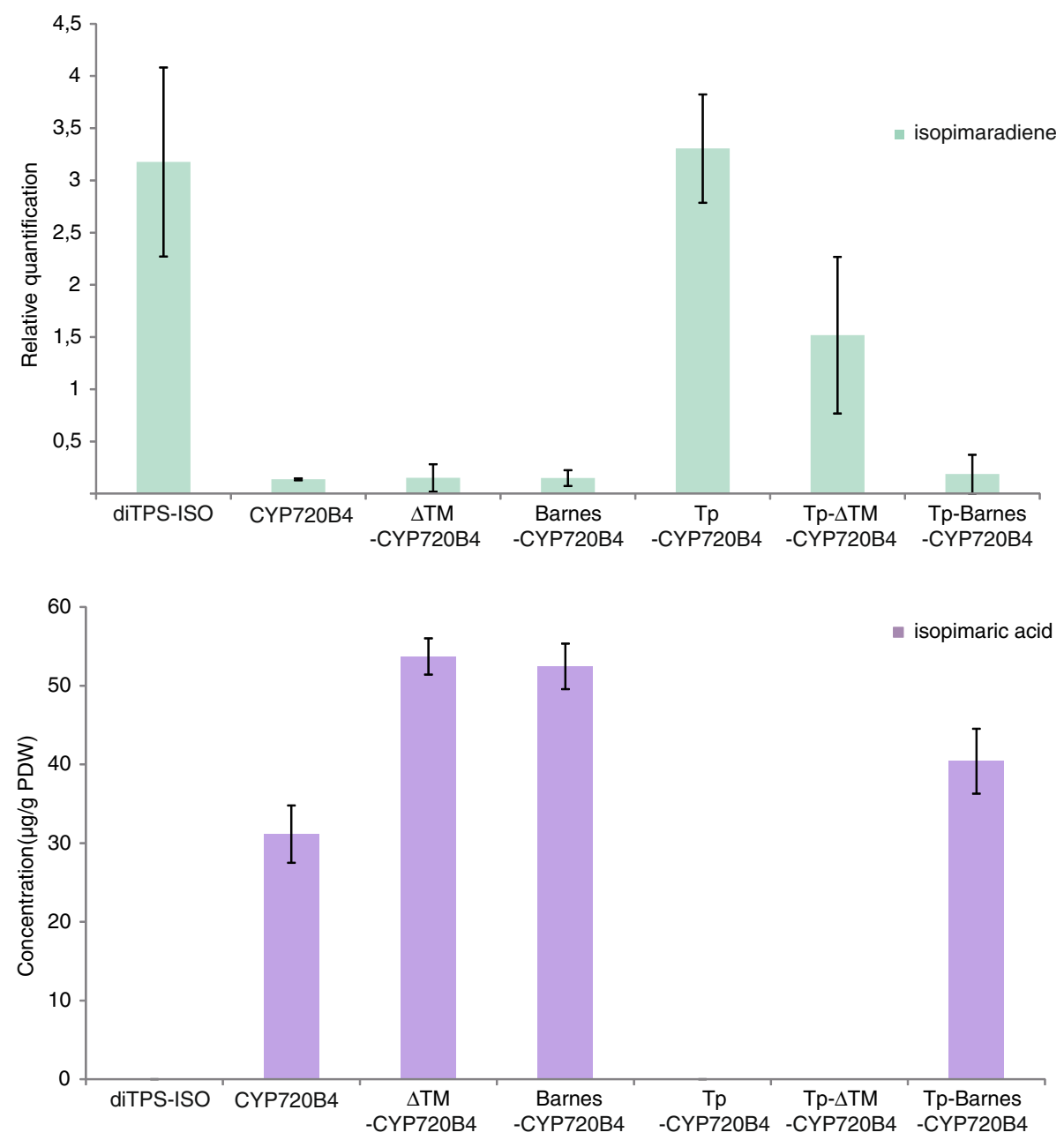

Fig. 6 Quantification of $\mathbf{a}$ isopimaradiene and $\mathbf{b}$ isopimaric acid detected in infiltrated leaves. The bar labelled (diTPS-ISO) indicates the quantities of isopimaradiene/isopimaric acid produced upon co-expressing diTPS-ISO, DXS and GGPPS constructs. All other bars display the quantities of isopimaradiene/isopimaric acid produced upon co-expressing different CYP constructs with DXS, GGPPS and diTPS-ISO. Isopimaradiene is quantified relatively to the internal standard eicosene, and the isopimaric acid quantity was quantified relative to plant dry weight (PDW). Biological triplicates were used for the quantification

transformed via electroporation as described in [51]. Positive transformants were selected on YEP plates with $50 \mathrm{~g} /$ $\mathrm{L}$ kanamycin and $25 \mathrm{~g} / \mathrm{L}$ rifampicin, incubated at $28^{\circ} \mathrm{C}$ for two days. $N$. benthamiana plants were grown in greenhouse conditions (Day temperature $25{ }^{\circ} \mathrm{C}$; Night temperature $20^{\circ} \mathrm{C} ; 16 \mathrm{~h}$ light $/ 8 \mathrm{~h}$ dark cycle; light intensity of $200-600 \mu \mathrm{mol}$ photons $\left.. \mathrm{m}^{-2} . \mathrm{s}^{-1}\right)$ in peat and were infiltrated with transformed $A$. tumefaciens as described in [15]. The infiltrated plants grew in the greenhouse for four days, followed by harvesting of leaf material for further analysis.

\section{Sequences and constructs}

The P19 silencing repressor gene [GenBank: NP_612584.1] [29] was cloned into pCAMBIA1300 vector (pLIFE-33) and the diTPS-ISO gene from Picea abies
[GenBank: AY473620.2] was cloned with a C-terminal FLAG tag into pEAQ-HT vector [52]. The DXS and GGPPs [GenBank: KP889114 and KP889115 respectively] coding regions from $P$. barbatus were also inserted into the pCAMBIA vector. CYP720B4 coding sequence from Picea sitchensis [GenBank: HM245403.1], Barnes$\mathrm{CYP}$ and $\triangle \mathrm{Tm}-\mathrm{CYP}$ coding sequences were inserted into an AgeI-XhoI linearized pEAQ-HT vector via T4 ligation (T4 ligase from New England Biolabs). The transit peptide ( $\mathrm{Tp}$ ) sequence coding for the N-terminal 52 amino acid of the Arabidopsis ferredoxin protein FedA [GenBank: AY128936] was amplified from the template Fd-CYP79A1 construct in pEAQ-HT mentioned in [15] and was introduced upstream of the CYP720B4 coding sequences using overlap-extension PCR [53]. The primers used in this work are listed in Table 1. 
Table 1 PCR primers

\begin{tabular}{lll}
\hline Name & Sequence $\left(5^{\prime}\right.$-3') & Comment \\
\hline P1 & TCGCGACCGGTAATGGCGCCCATGGC & CYP720B4 forward primer \\
P2 & ATCTCGAGTATTCATTCTCTACTCTACCATGAAG & CYP720B4 reverse primer \\
P3 & GCGACCGGTAATGGCTCTGTTATTAGC & Barnes-CYP720B4 forward primer \\
P4 & TCGCGACCGGTAATGAGTAATAAGGAGGT & $\Delta$ Tm-CYP720B4 forward primer \\
P5 & CGACCGGTAATGGCTTCCACTGCTC & Tp forward primer \\
P6 & AGCGGCTGTGACACG & Tp reverse primer \\
P7 & TCGTCTTTGTAGTCTTCATTCTCTACTCTAC & CYP720B4 reverse primer, containing FLAG tag \\
\hline
\end{tabular}

\section{GC-MS analysis}

For GC-MS analysis, four leaf discs $(\varnothing=3 \mathrm{~cm})$ from $N$. benthamiana plants infiltrated with the respective constructs were taken. The cut leaves were incubated in $1.5 \mathrm{ml}$ glass vials containing pure hexane (GC-MS grade) spiked with the internal standard $(0.1 \mathrm{mg} / \mathrm{L}$ eicosene) for $1 \mathrm{~h}$ at room temperature at the Roto-Shake Genie ${ }^{\oplus}$ (Scientific Industries, Inc.) revolving at 25-30 cycles/min.

Extracts were analyzed on a Shimadzu GCMS-QP2010 Ultra using an HP-5MS column (20 m x $0.180 \mathrm{~mm}$ i.d., $0.18 \mu \mathrm{m}$ film thickness, Agilent technologies). Injection volume and temperature were set at $1 \mu \mathrm{L}$ and $250{ }^{\circ} \mathrm{C}$ in splitless mode (GC program: $60{ }^{\circ} \mathrm{C}$ for $1 \mathrm{~min}$, ramp at rate $20{ }^{\circ} \mathrm{C}$ min- 1 to $160{ }^{\circ} \mathrm{C}$, ramp at rate $5{ }^{\circ} \mathrm{C}$ min- 1 to $240{ }^{\circ} \mathrm{C}$, ramp at rate $20{ }^{\circ} \mathrm{C}$ min- 1 to $320{ }^{\circ} \mathrm{C}$ and hold for $2 \mathrm{~min}) . \mathrm{H}_{2}$ were used as carrier gas with a linear velocity at $66.5 \mathrm{~cm} \mathrm{~s}-1$ and a purge flow of $4 \mathrm{~mL} \mathrm{min-1}$ for $1 \mathrm{~min}$. Ion source temperature of the mass spectrometer was set to $280{ }^{\circ} \mathrm{C}$ and spectra's was recorded from $\mathrm{m} / \mathrm{z}$ 50 to $\mathrm{m} / \mathrm{z} 350$ with a solvent cutoff at $4 \mathrm{~min}$. Compound identification was done with comparison to authentic standard of isopimaradiene as described in [26].

Isopimaradiene was quantified on a SCION 436 GCFID (Bruker). $2 \mu \mathrm{L}$ sample was injected in splitless mode at $280 \mathrm{C}^{\circ}$ (GC program same to the one mentioned above). After the transfer of the sample from the injection port to the HP5 column (1 min), the injection port was set to split 1:50. $\mathrm{H}_{2}$ was used as carrier gas with a constant flow of $1 \mathrm{~mL}$ min-1. The FID was set at $300{ }^{\circ} \mathrm{C}$, with a $\mathrm{N}_{2}$ flow of $25 \mathrm{~mL}$ min- $1, \mathrm{H}_{2}$ at $30 \mathrm{~mL} \mathrm{~min}-1$ and air $300 \mathrm{~mL} \mathrm{min-1.} \mathrm{Data} \mathrm{sampling} \mathrm{rate} \mathrm{was} \mathrm{taken} \mathrm{at} \mathrm{a} \mathrm{fre-}$ quency of 10 Hertz. Isopimaradiene was identified based on the retention time compared to the authentic standard. Quantification of isopimaradiene was done by integration of the isopimaradiene peak area and calculated from the concentration of the internal standard. The relative response factor between isopimaradiene and the internal standard was set to 1 .

\section{LC-MS analysis}

Four leaf discs per plant were cut, flash-frozen in liquid nitrogen, and homogenized in $80 \mathrm{v} / \mathrm{v} \%$ methanol aqueous solution. Samples were diluted with water to $50 \mathrm{v} / \mathrm{v} \%$ methanol final concentration and briefly spun down in mini-centrifuge. $40 \mu \mathrm{L}$ of the supernatant was used for LC-MS analysis. The LC-MS analysis was performed as previously described [54] with the gradient program set as: 0 to $1 \mathrm{~min}$, isocratic $50 \% \mathrm{~B}$; 1 to $10 \mathrm{~min}$, linear gradient 50 to $95 \% \mathrm{~B} ; 10$ to $11.4 \mathrm{~min}$, isocratic $98 \% \mathrm{~B} ; 11.4$ to $17 \mathrm{~min}$, isocratic $50 \% \mathrm{~B}$.

\section{Pigment analysis}

The pigments composition of leaves was analyzed immediately after extraction in acetone $/ \mathrm{H}_{2} \mathrm{O} / 25 \% \mathrm{NH}_{4} \mathrm{OH}$ $(80 / 20 / 1 \mathrm{vol} / \mathrm{vol} / \mathrm{vol})$ as described in $[55,56]$ with minor modifications. The Dionex HPLC system with a Supelco LICHROSPHER RP18-5 analytical (250 mm x $4.6 \mathrm{~mm}$ inner diameter; $5 \mu \mathrm{m}$ particle size) was used. The mobile phase consisted of two solvents, A: acetonitrile/methanol/0.1 M Tris-HCl pH 8.0 (84/2/14 vol/vol/vol) and B: methanol/ethyl acetate $(68 / 32 \mathrm{vol} / \mathrm{vol})$. The pigments were eluted with a linear gradient from $100 \%$ solvent A to $100 \%$ solvent B over $12 \mathrm{~min}$, followed by an isocratic elution with $100 \%$ solvent B for $6 \mathrm{~min}$, and a linear gradient of $100 \%$ solvent B to $100 \%$ solvent A in $1 \mathrm{~min}$. The column was regenerated with $100 \%$ solvent A for $11 \mathrm{~min}$ before injection of the next sample. Injection volume was $40 \mu \mathrm{l}$, the flow rate was $1 \mathrm{ml} / \mathrm{min}$, and peaks were detected and integrated at $445 \mathrm{~nm}$ for carotenoid and chlorophyll content. Pigments were identified by comparing retention times and absorption spectra with standard pigments (DHI, Hørsholm, Denmark). Quantification was performed by integration of the elution peaks at $445 \mathrm{~nm}$ using the program Chromeleon version 6.7 (Dionex, Sunnyvale, CA).

\section{Immunoblotting}

$10 \mathrm{mg}$ of flash-frozen leaf material was grinded using a ball mill (Retsch, US) (Pulse 30 and $1.5 \mathrm{~min}, 3$ times). To the crushed leaf material $100 \mu \mathrm{L}$ of SDS-PAGE sample buffer (Bio-Rad) was added and vortexed for 2 mins. The vortexed mixture was centrifuged at $10000 \mathrm{~g}$ for 10 mins. The supernatant was recovered and used for gel electrophoresis on $12 \%$ SDS Criterion ${ }^{\mathrm{Ts}}$ TGX Stain- 
Free $^{\text {in }}$ Precast Gel (Bio-Rad). Immunoblotting was performed as described in [15]. Proteins were transferred to PVDF membrane using the Trans-Blot ${ }^{\oplus}$ Turbo $^{\text {ma }}$ Transfer Starter System (Bio-Rad) according to manufacturer's protocol. The primary antibodies used were monoclonal anti-FLAG M2 antibody (Sigma-Aldrich Cat\# F3165, RRID: AB_259529) of 1:1000 dilution in $5 \%$ skimmed milk PBS-T. As the secondary antibody, polyclonal rabbit anti-mouse/HRP (DAKO Cat\# P 0447) of 1:2000 dilutions in $5 \%$ skimmed milk PBS-T was used. Gels and membranes were visualized in the ChemiDoc ${ }^{\mathrm{mx}}$ MP System (Bio-Rad). Total chlorophyll and chlorophyll $\mathrm{a} / \mathrm{b}$ ratios were determined in $80 \%$ acetone as described in [57].

\section{Abbreviations}

DXS: 1-deoxyxylulose-5-phosphate synthase; ER: Endoplasmic reticulum; GC-MS: Gas chromatography/mass spectrometry; GGPP: Geranylgeranyl diphosphate; GGPPs: GGPP synthase; HPLC: High performance liquid chromatography; LC-MS: liquid chromatography/mass spectrometry.

\section{Competing interests}

The authors declare that they have no competing interests.

\section{Authors' contributions}

$\mathrm{TG}, \mathrm{KV}, \mathrm{AZN}, \mathrm{BH}$ and PEJ conceived the study and planned the experiments. $\mathrm{TG}$ and $\mathrm{KV}$ performed all the experiments, data analysis and wrote the paper. JAR performed the GC-MS analysis. CEO performed the LC-MS analysis. All authors read and approved the final manuscript.

\section{Acknowledgements}

The authors would like to thank Dr. Lars B. Scharff and Silas Busck Mellor for fruitful discussions, and the green house service personnel for their assistance (University of Copenhagen, Faculty of Science, Department of Plant and Environmental Sciences). We are grateful to Dr. Søren Spanner Bach and Anna Holzwarth for the pCAMBIA1300 vector (pLIFE-33) and the FLAG-tagged diTPS-ISO construct respectively. This study was financially supported by (1) the VILLUM Center of Excellence "Plant Plasticity", (2) the "Center of Synthetic Biology" funded by the UNIK research initiative of the Danish Ministry of Science, Technology and Innovation, (3) "bioSYNergy" funded by the UCPH Excellence Programme for Interdisciplinary Research, and (4) "Plant Power: Light-Driven Synthesis of Complex Terpenoids Using Cytochromes P450" (12-131834) funded by Innovation Fund Denmark (previously the Danish Council for Strategic Research), Program Commission on Strategic Growth Technologies.

\section{Author details}

'Department of Plant and Environmental Sciences, Copenhagen Plant Science Centre, UNIK Center for Synthetic Biology, Villum Research Center "Plant Plasticity", University of Copenhagen, Thorvaldsensvej 40, DK-1871 Frederiksberg C, Copenhagen, Denmark. ${ }^{2}$ Present address: Plant and Microbial Biology, University of California, 371 Koshland Hall, Berkeley, CA 94720, USA.

Received: 10 November 2015 Accepted: 7 December 2015 Published online: 22 December 2015

\section{References}

1. Hartmann T. From waste products to ecochemicals: Fifty years research of plant secondary metabolism. Phytochemistry. 2007;68(22-24):2831-46. doi:10.1016/j.phytochem.2007.09.017

2. Zi J, Mafu S, Peters RJ. To gibberellins and beyond! Surveying the evolution of (di)terpenoid metabolism. Annu Rev Plant Biol. 2014;65:259-86. doi:10.1146/annurev-arplant-050213-035705.

3. Lassen LM, Nielsen AZ, Ziersen B, Gnanasekaran T, Moller BL, Jensen PE. Redirecting photosynthetic electron flow into light-driven synthesis of alternative products including high-value bioactive natural compounds. ACS Synth Biol. 2014;3(1):1-12.

4. Staniek A, Bouwmeester H, Fraser PD, Kayser O, Martens S, Tissier A, et al. Natural products - modifying metabolite pathways in plants. Biotechnol J. 2013;8(10):1159-71. doi:10.1002/biot.201300224.

5. Zerbe P, Bohlmann J. Plant diterpene synthases: exploring modularity and metabolic diversity for bioengineering. Trends Biotechnol. 2015;33(7):419-28. doi:10.1016/j.tibtech.2015.04.006

6. Zerbe P, Bohlmann J. Enzymes for synthetic biology of ambroxide-related diterpenoid fragrance compounds. Adv Biochem Eng Biotechnol. 2015;148:427-47. doi:10.1007/10_2015_308.

7. Tholl $\mathrm{D}$. Terpene synthases and the regulation, diversity and biological roles of terpene metabolism. Curr Opin Plant Biol. 2006;9(3):297-304. doi:10.1016/j.pbi.2006.03.014

8. Hamberger B, Bak S. Plant P450s as versatile drivers for evolution of speciesspecific chemical diversity. Philos Trans R Soc Lond B Biol Sci. 2013; 368(1612):20120426. doi:10.1098/rstb.2012.0426.

9. Munro AW, Girvan HM, Mason AE, Dunford AJ, McLean KJ. What makes a P450 tick? Trends Biochem Sci. 2013;38(3):140-50. doi:10.1016/j.tibs.2012.11.006.

10. Poulos T, Johnson E. Structures of Cytochrome P450 Enzymes. In: Ortiz de Montellano P, editor. Cytochrome P450. US: Springer; 2005. p. 87-114.

11. Froehlich JE, Itoh A, Howe GA. Tomato Allene Oxide Synthase and Fatty Acid Hydroperoxide Lyase, Two Cytochrome P450s Involved in Oxylipin Metabolism, Are Targeted to Different Membranes of Chloroplast Envelope. Plant Physiol. 2001;125(1):306-17. doi:10.1104/pp.125.1.306.

12. Pateraki I, Heskes AM, Hamberger B. Cytochromes P450 for Terpene Functionalisation and Metabolic Engineering. Adv Biochem Eng Biotechnol. 2015;148:107-39. doi:10.1007/10_2014_301.

13. O'Reilly E, Kohler V, Flitsch SL, Turner NJ. Cytochromes P450 as useful biocatalysts: addressing the limitations. Chem Commun (Camb). 2011:47(9):2490-501. doi:10.1039/c0cc03165h.

14. Jensen K, Jensen PE, Moller BL. Light-driven cytochrome p450 hydroxylations. ACS Chem Biol. 2011;6(6):533-9. doi:10.1021/cb100393j.

15. Nielsen AZ, Ziersen B, Jensen $K$, Lassen LM, Olsen CE, Moller BL, et al. Redirecting Photosynthetic Reducing Power toward Bioactive Natural Product Synthesis. ACS Synth Biol. 2013;2(6):308-15. doi:10.1021/sb300128r.

16. Jensen K, Jensen PE, Moller BL. Light-driven chemical synthesis. Trends Plant Sci. 2012;17(2):60-3. doi:10.1016/j.tplants.2011.12.008.

17. Lassen LM, Nielsen AZ, Olsen CE, Bialek W, Jensen K, Moller BL, et al. Anchoring a plant cytochrome P450 via PsaM to the thylakoids in Synechococcus sp. PCC 7002: evidence for light-driven biosynthesis. PLoS ONE. 2014;9(7):e102184. doi:10.1371/journal.pone.0102184.

18. Gangl D, Zedler JA, Wlodarczyk A, Jensen PE, Purton S, Robinson C. Expression and membrane-targeting of an active plant cytochrome P450 in the chloroplast of the green alga Chlamydomonas reinhardtii. Phytochemistry. 2015;110:22-8. doi:10.1016/j.phytochem.2014.12.006.

19. Wlodarczyk A, Gnanasekaran T, Nielsen AZ, Zulu NN, Mellor SB, Luckner M, et al. Metabolic engineering of light-driven cytochrome P450 dependent pathways into Synechocystis sp. PCC 6803. Metab Eng. 2016;33:1-11. doi:10.1016/j.ymben.2015.10.009.

20. Kopper BJ, Illman BL, Kersten PJ, Klepzig KD, Raffa KF. Effects of Diterpene Acids on Components of a Conifer Bark Beetle-Fungal Interaction: Tolerance by Ips pini and Sensitivity by Its Associate Ophiostoma ips. Environ Entomol. 2005;34(2):486-93. doi:10.1603/0046-225x-34.2.486.

21. Powell JS, Raffa KF. Effects of Selected Larix laricina Terpenoids on Lymantria dispar (Lepidoptera: Lymantriidae) Development and Behavior. Environ Entomol. 1999;28(2):148-54. doi:10.1093/ee/28.2.148.

22. Wagner M, Benjamin D, Clancy K, Schuh B. Influence of diterpene resin acids on feeding and growth of larch sawfly, Pristiphora erichsonii (Hartig). J Chem Ecol. 1983;9(1):119-27. doi:10.1007/BF00987776.

23. Smith E, Williamson E, Zloh M, Gibbons S. Isopimaric acid from Pinus nigra shows activity against multidrug-resistant and EMRSA strains of Staphylococcus aureus. Phytother Res. 2005;19(6):538-42. doi:10.1002/ptr.1711.

24. Keeling $\mathrm{Cl}$, Bohlmann J. Diterpene resin acids in conifers. Phytochemistry. 2006;67(22):2415-23. doi:10.1016/.jphytochem.2006.08.019.

25. Keeling Cl, Weisshaar S, Lin RP, Bohlmann J. Functional plasticity of paralogous diterpene synthases involved in conifer defense. Proc Natl Acad Sci U S A. 2008;105(3):1085-90. doi:10.1073/pnas.0709466105.

26. Hamberger B, Ohnishi T, Hamberger B, Seguin A, Bohlmann J. Evolution of diterpene metabolism: Sitka spruce CYP720B4 catalyzes multiple oxidations 
in resin acid biosynthesis of conifer defense against insects. Plant Physiol. 2011;157(4):1677-95. doi:10.1104/pp.111.185843.

27. Jarvis P, Lopez-Juez E. Biogenesis and homeostasis of chloroplasts and other plastids. Nat Rev Mol Cell Biol. 2013;14(12):787-802. doi:10.1038/nrm3702.

28. Jensen $\mathrm{PE}$, Leister $\mathrm{D}$. Chloroplast evolution, structure and functions. F1000Prime Rep. 2014;6:40. doi:10.12703/p6-40.

29. Voinnet $O$, Rivas $S$, Mestre P, Baulcombe D. An enhanced transient expression system in plants based on suppression of gene silencing by the p19 protein of tomato bushy stunt virus. Plant J. 2003;33(5):949-56. doi:10.1046/j.1365-313X.2003.01676.X.

30. Brückner K, Tissier A. High-level diterpene production by transient expression in Nicotiana benthamiana. Plant Methods. 2013;9(1):46 doi:10.1186/1746-4811-9-46.

31. Englund E, Andersen-Ranberg J, Miao R, Hamberger B, Lindberg P. Metabolic Engineering of Synechocystis sp. PCC 6803 for Production of the Plant Diterpenoid Manoyl Oxide. ACS Synth Biol. 2015. doi:10.1021/ acssynbio.5b00070.

32. Vranova E, Coman D, Gruissem W. Network analysis of the MVA and MEP pathways for isoprenoid synthesis. Annu Rev Plant Biol. 2013;64:665-700. doi:10.1146/annurev-arplant-050312-120116.

33. Wright LP, Rohwer JM, Ghirardo A, Hammerbacher A, Ortiz-Alcaide M, Raguschke B, et al. Deoxyxylulose 5-Phosphate Synthase Controls Flux through the Methylerythritol 4-Phosphate Pathway in Arabidopsis. Plant Physiol. 2014;165(4):1488-504. doi:10.1104/pp.114.245191.

34. Pokhilko A, Bou-Torrent J, Pulido P, Rodriguez-Concepcion M, Ebenhoh O. Mathematical modelling of the diurnal regulation of the MEP pathway in Arabidopsis. New Phytol. 2015;206(3):1075-85. doi:10.1111/nph.13258.

35. Barnes HJ, Arlotto MP, Waterman MR. Expression and enzymatic activity of recombinant cytochrome P450 17 alpha-hydroxylase in Escherichia coli. Proc Natl Acad Sci U S A. 1991:88(13):5597-601.

36. Smeekens S, Bauerle C, Hageman J, Keegstra K, Weisbeek P. The role of the transit peptide in the routing of precursors toward different chloroplast compartments. Cell. 1986;46(3):365-75. doi:10.1016/0092-8674(86)90657-4.

37. Lee DW, Jung C, Hwang I. Cytosolic events involved in chloroplast protein targeting. Biochim Biophys Acta, Mol Cell Res. 2013;1833(2):245-52. doi:10.1016/j.bbamcr.2012.03.006.

38. Pernecky SJ, Larson JR, Philpot RM, Coon MJ. Expression of truncated forms of liver microsomal P450 cytochromes 2B4 and 2E1 in Escherichia coli: influence of NH2-terminal region on localization in cytosol and membranes. Proc Natl Acad Sci U S A. 1993;90(7):2651-5.

39. Denisov IG, Shih AY, Sligar SG. Structural differences between soluble and membrane bound cytochrome P450s. J Inorg Biochem. 2012;108:150-8. doi:10.1016/j.jinorgbio.2011.11.026.

40. Hasunuma T, Miyazawa S-I, Yoshimura S, Shinzaki Y, Tomizawa K-I, Shindo K, et al. Biosynthesis of astaxanthin in tobacco leaves by transplastomic engineering. Plant J. 2008;55(5):857-68. doi:10.1111/j.1365-313X.2008.03559.x.

41. Apel W, Bock R. Enhancement of carotenoid biosynthesis in transplastomic tomatoes by induced lycopene-to-provitamin A conversion. Plant Physiol. 2009:151(1):59-66. doi:10.1104/pp.109.140533.

42. Maliga P, Bock R. Plastid biotechnology: food, fuel, and medicine for the 21st century. Plant Physiol. 2011;155(4):1501-10. doi:10.1104/pp.110.170969.

43. Scharff LB, Bock R. Synthetic biology in plastids. Plant J. 2014;78(5):783-98. doi:10.1111/tpj.12356.

44. Gangl D, Zedler JAZ, Rajakumar PD, Martinez EMR, Riseley A, Włodarczyk A et al. Biotechnological exploitation of microalgae. J Exp Bot. 2015. doi:10.1093/jxb/erv426.

45. Bernhardt R. Cytochromes P450 as versatile biocatalysts. J Biotechnol. 2006;124(1):128-45. doi:10.1016/j.jbiotec.2006.01.026.

46. Ignea C, loannou E, Georgantea P, Loupassaki S, Trikka FA, Kanellis AK, et al. Reconstructing the chemical diversity of labdane-type diterpene biosynthesis in yeast. Metab Eng. 2015;28:91-103. doi:10.1016/j.ymben.2014.12.001.

47. Podust LM, Sherman DH. Diversity of P450 enzymes in the biosynthesis of natural products. Nat Prod Rep. 2012;29(10):1251-66. doi:10.1039/ c2np20020a.

48. Zhao YJ, Cheng QQ, Su P, Chen X, Wang XJ, Gao W, et al. Research progress relating to the role of cytochrome $\mathrm{P} 450$ in the biosynthesis of terpenoids in medicinal plants. Appl Microbiol Biotechnol. 2014;98(6):2371-83. doi:10.1007/s00253-013-5496-3

49. Paddon CJ, Westfall PJ, Pitera DJ, Benjamin K, Fisher K, McPhee D, et al. High-level semi-synthetic production of the potent antimalarial artemisinin. Nature. 2013;496(7446):528-32. doi:10.1038/nature12051.
50. Ajikumar PK, Xiao WH, Tyo KE, Wang Y, Simeon F, Leonard E, et al. Isoprenoid pathway optimization for Taxol precursor overproduction in Escherichia coli. Science. 2010;330(6000):70-4. doi:10.1126/science.1191652.

51. Haldrup A, Lunde C, Scheller HV. Arabidopsis thaliana plants lacking the PSID subunit of photosystem I suffer severe photoinhibition, have unstable photosystem I complexes, and altered redox homeostasis in the chloroplast stroma. J Biol Chem. 2003;278(35):33276-83. doi:10.1074/jbc.M305106200.

52. Sainsbury F, Thuenemann EC, Lomonossoff GP. pEAQ: versatile expression vectors for easy and quick transient expression of heterologous proteins in plants. Plant Biotechnol J. 2009;7(7):682-93. doi:10.1111/j.1467-7652.2009. 00434.X.

53. Higuchi R, Krummel B, Saiki RK. A general method of in vitro preparation and specific mutagenesis of DNA fragments: study of protein and DNA interactions. Nucleic Acids Res. 1988;16(15):7351-67. doi:10.1093/nar/16.15.7351.

54. Takos A, Lai D, Mikkelsen L, Abou Hachem M, Shelton D, Motawia MS, et al. Genetic Screening Identifies Cyanogenesis-Deficient Mutants of Lotus japonicus and Reveals Enzymatic Specificity in Hydroxynitrile Glucoside Metabolism. Plant Cell. 2010;22(5):1605-19. doi:10.1105/tpc.109.073502.

55. Jensen PE, Rosgaard L, Knoetzel J, Scheller HV. Photosystem I activity is increased in the absence of the PSI-G subunit. J Biol Chem. 2002;277(4): 2798-803. doi:10.1074/jbc.M110448200.

56. Garcia-Plazaola Jl, Matsubara S, Nicotra A. Determination of chlorophylls and carotenoids by HPLC PrometheusWiki. 2012. http://prometheuswiki.publish. csiro.au/tiki-pagehistory.php?page=Determination\%20 f\%20chlorophylls\%20 and\%20carotenoids\%20by\%20HPLC\&preview=10. Accessed 2015-08-07 07: 07:26 UTC 2015

57. Lichtenthaler HK. Chlorophylls and carotenoids: Pigments of photosynthetic biomembranes. In: Lester Packer RD, editor. Methods in Enzymology. Massachusetts: Academic; 1987. p. 350-82.

\section{Submit your next manuscript to BioMed Central and we will help you at every step:}

- We accept pre-submission inquiries

- Our selector tool helps you to find the most relevant journal

- We provide round the clock customer support

- Convenient online submission

- Thorough peer review

- Inclusion in PubMed and all major indexing services

- Maximum visibility for your research

Submit your manuscript at www.biomedcentral.com/submit
( ) BioMed Central 